\title{
What Happened? Exploring the Relation between Traumatic Stress and Provisional Mental Health Diagnoses for Children and Youth
}

\author{
Kim Arbeau1, Laura Theall1, Keith Willoughby'1, Jared M. J. Berman ${ }^{1,2}$, Shannon L. Stewart ${ }^{2}$ \\ ${ }^{1}$ Child and Parent Resource Institute, London, Ontario, Canada \\ ${ }^{2}$ Western University, London, Ontario, Canada \\ Email: kim.arbeau@ontario.ca
}

How to cite this paper: Arbeau, K., Theall, L., Willoughby, K., Berman, J. M. J., \& Stewart, S. L. (2017). What Happened? Exploring the Relation between Traumatic Stress and Provisional Mental Health Diagnoses for Children and Youth. Psychology, 8, 2485-2495.

https://doi.org/10.4236/psych.2017.814157

Received: October 11, 2017

Accepted: December 18, 2017

Published: December 21, 2017

Copyright $\odot 2017$ by authors and Scientific Research Publishing Inc. This work is licensed under the Creative Commons Attribution International License (CC BY 4.0).

http://creativecommons.org/licenses/by/4.0/

\begin{abstract}
Objective: Traumatic stress can impact behaviours and neurological functioning of children and youth, with symptoms appearing similar to behaviours associated with psychiatric diagnoses (Siegfried et al., 2016). This study sought to examine the link between provisional diagnoses and trauma in a sample of children/youth receiving mental health services. Methods: A sample of 6649 children/youth (59\% males) aged $4-18$ years $\left(M_{\text {age }}=11.99, S D=3.57\right)$ receiving services from 45 mental health agencies in Ontario were assessed using the interRAI Child and Youth Mental Health (ChYMH) instrument (Stewart et al., 2015a). We examined the interRAI Traumatic Life Events Collaborative Action Plan (CAP; Stewart et al., 2015b) and provisional diagnoses of attention-deficit/hyperactivity disorder (ADHD), anxiety disorders, reactive attachment disorder (RAD), mood disorders, substance-related disorders, and sleep disorders. Results: Compared to boys, girls were more likely to trigger the interRAI Traumatic Life Events CAP and to have a provisional diagnosis of anxiety, mood, and sleep disorders. Boys were more likely to have a provisional diagnosis of ADHD than girls. Multiple logistic regression analyses indicated that boys diagnosed with substance-related disorders had 1.79 higher odds of triggering the interRAI Traumatic Life Events CAP. ADHD, anxiety disorders, RAD, and mood disorders were also each significant predictors of potential traumatic stress regardless of sex. Conclusions/Implications: Findings suggest that several provisional diagnoses were significantly related to potential traumatic stress. Clinicians may find value in assessing for trauma, asking the question "What happened?" when confirming a psychiatric diagnosis in order to determine the best plan of care.
\end{abstract}




\section{Keywords}

Trauma, Adverse Childhood Experiences, interRAI, Developmental Trauma Disorder

\section{Introduction}

"What happened?" This simple question can have a significant impact on care planning and outcomes for children and youth who have been affected by traumatic experiences. The Adverse Childhood Experiences (ACE) Study is an extensive retrospective epidemiological study that assesses the effect of child abuse and family dysfunction on a variety of outcomes from adolescence to adulthood. Results from the ACE Study have demonstrated that exposure to adverse experiences (e.g., physical or sexual abuse, neglect, parental incarceration or mental illness) in childhood is strongly related to health risks in adulthood (e.g., adoption of high risk behaviours, depression, substance abuse disorders), with cumulative adverse events leading to increasing risk of certain chronic health problems in adulthood (e.g., heart disease, cancer, stroke, liver disease, chronic obstructive pulmonary disease) (Anda et al., 2008; Dube, Felitti, Dong, Giles, \& Anda, 2003; Felitti et al., 1998). Higher ACE scores have also been found to be related to earlier age of first arrest (Baglivio, Wolff, Piquero, \& Epps, 2015).

While the relation between experiences of early trauma or adversity and adult health and behavioural outcomes is now fairly well established (e.g., Caspi et al., 2016; Dube et al., 2003; Felitti et al., 1998), the impact of early trauma and adverse experiences throughout the course of childhood is less well understood (Perry, 1994). Exposure to trauma and adverse experiences leads to activation of a number of well-established physiological responses including a general discharge of the parasympathetic nervous system termed the acute stress response (Cannon, 1929; Koren, Arnon, \& Klein, 1999). While this response is generally adaptive in that it helps prepare the body to respond in the moment to a specific stressor or threat, there is significant evidence that chronic activation of this stress response during childhood can have a disruptive effect on a number of aspects of typical development, negatively impacting upon the development of attachments, sensorimotor skills, affect regulation, impulse control, and higher order cognitive skills (Cook et al., 2005; Shonkoff et al., 2012).

Chronically traumatized children can experience a wide variety of symptoms such as: amnesia, hypermnesia, dissociation, nightmares, flashbacks, school difficulties, difficulties in attention regulation, hyperactivity, irritability, feelings of fear and helplessness, and hypervigilance (Cook et al., 2005; Siegfried, Blackshear, \& The National Child Traumatic Stress Network, with assistance from the National Resource Center on ADHD, 2016; van der Kolk, 2005). Resultantly, children and youth exposed to significant levels of trauma, maltreatment, or ad- 
versity often meet the Diagnostic and Statistical Manual for Mental Disorders (DSM-5; American Psychiatric Association, 2013) criteria for a number of diagnoses, including: oppositional defiant disorder (ODD), attention-deficit/hyperactivity disorder (ADHD), conduct disorder, anxiety disorders, communication disorders, and reactive attachment disorder (RAD), with each possible diagnosis only partially capturing the child/youth's full range of relational and self-regulatory impairments (Cook et al., 2005).

It has been suggested by an increasing number of authors that no psychiatric diagnosis currently exists in the DSM-5 that is capable of adequately capturing the pervasive impact of trauma on children (Blaustein et al., 2003; D'Andrea, Ford, Stolbach, Spinazzola, \& van der Kolk, 2012; van der Kolk, 2005). Most traumatized children do not meet the diagnostic criteria for posttraumatic stress disorder (PTSD), and this diagnosis does not adequately address the developmental effects of trauma on children (Blaustein et al., 2003; van der Kolk, 2005). A proposal has been forwarded by van der Kolk (2005) to add a provisional disorder termed Developmental Trauma Disorder to the DSM in order to more accurately capture the symptomology associated with developmental exposure to trauma and adversity. Lacking such a category however, current practice often results in children/youth being given a variety of comorbid diagnoses (e.g., ADHD, depression, generalized anxiety disorder, RAD), which may not reflect the true nature or etiology associated with their symptomology (D'Andrea et al., 2012). As noted earlier, these diagnoses describe an aspect of the child's experience but do not capture the array of complex and interrelated negative effects that trauma can have on children and youth (Blaustein et al., 2003). Moreover, such diagnoses may not provide the proper guidelines for prevention and intervention for traumatized children/youth. Consequently, clinicians may implement ineffective treatments (van der Kolk, 2005). Therefore, it is important for clinicians to ask, "What happened?" in the past regarding traumatic experiences in order to ensure comprehensive treatment planning.

\section{Current Study}

Childhood trauma can affect and disrupt functioning across the lifespan. The course of treatment for a psychiatric diagnosis that does not account for the experience of trauma may not be the most effective intervention when traumatic stress is present but unrecognized. For example, some psychiatric diagnoses are predominantly treated using medications and/or specialized behavioural treatments (e.g., ADHD). Although medication for some disorders (e.g., anxiety) may help reduce some symptoms of traumatic stress such as hyper-arousal, medications alone may not be sufficient to improve overall functioning in the long term (Stewart et al., 2015b). The current study sought to explore the link between child and adolescent psychiatric diagnoses and trauma in a large sample of children/youth receiving mental health services from agencies across the province of Ontario. 
Sex differences are also important to consider when looking at diagnoses in childhood. The extant research evidence suggests that girls are more likely to show symptoms of internalizing disorders (e.g., anxiety, depression) and boys more likely to exhibit behaviours indicative of externalizing disorders (e.g., ADHD, conduct disorder) (Achenbach, Howell, Quay, Conners, \& Bates, 1991; Dodge, Pettit, \& Bates, 1994; Keiley et al., 2003). As such, the current study also considered sex differences while exploring the relation between traumatic stress and childhood mental health disorders.

\section{Method}

\subsection{Participants}

The participants in this study were a sample of 6649 children and youth who were receiving services from 45 mental health agencies across Ontario. The majority of participants were male (59\%), with 11 children/youth reported as "other" for sex. Participants classified as "other" were excluded from the sex-specific analyses due to small sample size. Ages ranged from 4 to 18 years $\left(M_{\text {age }}=11.99\right.$, $S D=3.57)$. Ethics approval was obtained from the research ethics board at Western University.

\subsection{Instrument}

The participants were assessed using the interRAI Child and Youth Mental Health (ChYMH) instrument (Stewart et al., 2015a). The ChYMH is a multidisciplinary comprehensive assessment tool that includes over 400 data elements. Trained assessors complete the assessment through interviews with the child/youth and caregivers, and using other available information sources (e.g., teachers, other involved professionals, clinical documents).

The interRAI assessment system is unique in that certain items from the instruments combine as algorithms to identify individuals in need of interventions for specific issues. The algorithms trigger Collaborative Action Plans (CAPs) which are brief documents created to inform clinicians on best practice for issue-based care planning. The interRAI ChYMH CAPs were developed in collaboration with experts from around the world specializing in child and youth mental health care, and provide evidence-informed guidelines and intervention recommendations for improving functioning and quality of life (Stewart et al., 2015c).

\subsection{Procedure}

The initial ChYMH assessments, completed prior to or at the time of admission to mental health services, were used in the analyses (i.e., follow-up and discharge assessments were excluded). Potential for traumatic stress was measured using the interRAI Traumatic Life Events CAP (Stewart et al., 2015b). The Traumatic Life Events CAP provides guidelines to assist in immediate safety planning and intervention for children/youth with actual or suspected experience of trauma. The traumatic events included in the algorithm for triggering this CAP are: se- 
rious accident or physical impairment; death of a caregiver; lived in a war zone or an area of violent conflict (combatant or civilian); witness a severe accident, disaster, terrorism, violence, or abuse; victim of crime; sexual assault or abuse; physical assault or abuse; emotional abuse; chronic neglect (insufficient provision of food, clothing, shelter); bullying; abandoned by caregivers; witnessed domestic violence.

This CAP has two trigger levels: Level A is triggered for recent traumatic events that have occurred during the past week or current safety concerns expressed by the young person or others; Level B identifies children and youth who do not trigger for immediate safety concerns, but have experienced traumatic event(s) at any point in their lives, plus either describe the event(s) as evoking a sense of horror or are currently exhibiting two or more stress response behaviours (for example, re-enactment through play, unrealistic fears, intrusive thoughts, panic, hypervigilance, nightmares, or flat affect indicative of dissociation). For the purpose of this study Levels A and B were combined to indicate whether the CAP was triggered (coded " 1 ") or not triggered (coded " 0 ") for each participant.

An item on the ChYMH provides the opportunity for documenting up to four diagnoses (described as "provisional or actual" in the instrument) per child/youth as determined by a psychiatrist, attending physician, or psychologist. The use of "provisional" psychiatric diagnosis in childhood is common practice, as the stability of many psychiatric diagnoses throughout childhood and over time has yet to be established (Pettit, Morgan, \& Paukert, 2005). When using the ChYMH, assessors rank diagnoses according to how much each contributed to the current admission (i.e., $1=$ most important; 2 = second most important; 3 = third most important; 4 = less important). Those diagnostic categories not assigned are coded as " $0=$ not present". If no diagnosis is available, all categories are coded as " 8 ". The following provisional diagnoses were of interest in the current study: ADHD (e.g., predominantly inattentive type, predominantly hyperactive-impulsive type, or combined type), anxiety disorders (e.g., separation anxiety disorder, panic disorder, agoraphobia, social phobia, obsessive-compulsive disorder, post-traumatic stress disorder, acute stress disorder, generalized anxiety disorder, substance-induced anxiety disorder), RAD (e.g., inhibited or disinhibited form), mood disorders (e.g., major depressive disorder, bipolar disorder, mood disorder not otherwise specified, dysthymia, cyclothymia), substance-related disorders (e.g., alcohol use disorders, amphetamine-related disorders, caffeine-related disorders, cannabis-related disorders, cocaine-related disorders, hallucinogen-related disorders, inhalant-related disorders, nicotine-related disorders, opioid-related disorders, phencyclidine-related disorders, sedative-, hypnotic-, or anxiolytic-related disorders, polysubstance-related disorders), and sleep disorders (e.g., primary sleep disorders, dyssomnias, parasomnias). These variables were recoded for the analyses. Responses of 1 - 4 were reassigned a code of " 1 " to indicate "present", responses of " 0 " and " 8 " were recoded as" 0 " for "not present". 


\section{Results}

\subsection{Preliminary Data Analysis}

In the present study, $51 \%$ of the children/youth triggered the interRAI Traumatic Life Events CAP. Moreover, $40 \%$ had a provisional diagnosis of ADHD, 33\% were reported as having a provisional diagnosis in the category of anxiety disorders, $15 \%$ were reported as having a provisional diagnosis in the category of mood disorders, $3 \%$ were reported as having a provisional diagnosis in the category sleep disorders, $2 \%$ had a provisional diagnosis of $\mathrm{RAD}$, and $2 \%$ were reported as having a provisional diagnosis in the category substance-related disorders. Some sex differences were found. Girls were more likely to trigger the interRAI Traumatic Life Events CAP and to have provisional anxiety, mood, and sleep disorders than boys. Boys were more likely to have a provisional diagnosis of ADHD compared to girls (see Table 1).

Of those children/youth who had a provisional diagnosis of ADHD, 54\% triggered the interRAI Traumatic Life Events CAP. Of those children/youth who were reported as having a provisional diagnosis in the category anxiety disorders, $61 \%$ triggered the interRAI Traumatic Life Events CAP. Further, of those children/youth who had a provisional diagnosis of RAD, 75\% also triggered the interRAI Traumatic Life Events CAP. For those children/youth who had a provisional diagnosis in the category of mood disorders, $66 \%$ also triggered the interRAI Traumatic Life Events CAP. There were $68 \%$ of children/youth who had a provisional diagnosis in the category of substance-related disorders who also triggered the interRAI Traumatic Life Events CAP. Similarly, $66 \%$ of children/youth in this study had a provisional diagnosis in the category sleep disorders and triggered the interRAI Traumatic Life Events CAP.

Table 1. Examination of the differences between males and females on the interRAI Traumatic Life Events CAP and the provisional diagnostic categories.

\begin{tabular}{ccc}
\hline & \multicolumn{2}{c}{ Sex } \\
\cline { 2 - 3 } & Male $(\%)(n=3940)$ & Female $(\%)(n=2698)$ \\
\hline ADHD & $46.7_{\mathrm{a}}$ & $57.1_{\mathrm{b}}$ \\
Traumatic Life Events CAP & $50.4_{\mathrm{a}}$ & $25.4_{\mathrm{b}}$ \\
RAD & $29.7_{\mathrm{a}}$ & $38.3_{\mathrm{b}}$ \\
Mood Disorders & 2.4 & 1.9 \\
Substance-Related Disorders & $9.9_{\mathrm{a}}$ & $22.3_{\mathrm{b}}$ \\
Sleep Disorders & 2.3 & 2.4 \\
& $2.0_{\mathrm{a}}$ & $3.5_{\mathrm{b}}$
\end{tabular}

Note. Different subscripts equal significant differences between males and females at $p<0.05$ in the chi-square analyses. 


\subsection{Primary Data Analysis}

Two multiple logistic regression analyses were conducted to predict triggering of the interRAI Traumatic Life Events CAP from the following provisional diagnostic categories: ADHD, anxiety disorders, RAD, mood disorders, substance-related disorders and sleep disorders. A multiple logistic regression analysis was run separately for boys and girls. For boys, the full model provided a significantly better fit to the data than the constant-only model, which indicates that the predictors sufficiently distinguish between those children/youth who triggered the interRAI Traumatic Life Events CAP from those who did not trigger this CAP $(\chi 2=122.82, d f=6, p=0.000)$. A goodness of fit model was demonstrated by a non-statistically significant result on the Hosmer-Lemeshow test, $\chi^{2}(n=3940)=2.01, d f=3, p=0.570$. Results indicated that for boys, having a diagnosis of ADHD, anxiety disorders, RAD, mood disorders, and substance-related disorders increased the odds of triggering the interRAI Traumatic Life Events CAP. See Table 2 for the results from this model.

A second multiple logistic regression analysis was conducted for girls. For girls, again the full model provided a significantly better fit to the data than the constant-only model ( $\left.\chi^{2}=91.11, d f=6, p=0.000\right)$. A goodness of fit model was demonstrated by a non-statistically significant result on the Hosmer-Lemeshow test, $\chi^{2}(n=2698)=8.33, d f=4, p=0.080$. Similar to boys, the results indicated that for girls having a diagnosis of ADHD, anxiety disorders, RAD, and mood disorders increased the odds of triggering the interRAI Traumatic Life Events CAP. However, for girls, substance-related disorders were not a significant predictor in the model. See Table 3 for results of the model for girls.

Table 2. Multiple logistic regression analysis predicting the interRAI Traumatic Life Events CAP from provisional diagnoses for boys.

\begin{tabular}{cccccc}
\hline Predictor & $B$ & Wald & Odds ratio $\operatorname{Exp}(B)$ & $95 \%$ confidence interval & $p$ value \\
\hline ADHD & .26 & 15.08 & 1.29 & {$[1.14,1.47]$} & .000 \\
Anxiety Disorders & .46 & 38.63 & 1.59 & {$[1.37,1.83]$} & .000 \\
RAD & .87 & 13.27 & 2.38 & {$[1.49,3.78]$} & .000 \\
Mood Disorders & .29 & 6.54 & 1.34 & {$[1.07,1.67]$} & .011 \\
Substance-Related & .58 & 6.31 & 1.79 & {$[1.13,2.82]$} & .012 \\
Disorders & & & & & \\
Sleep Disorders & .39 & 2.54 & 1.47 & {$[.92,2.36]$} & .111 \\
\hline
\end{tabular}

Table 3. Multiple logistic regression analysis predicting the interRAI Traumatic Life Events CAP from provisional diagnoses for girls.

\begin{tabular}{cccccc}
\hline Predictor & $B$ & Wald & Odds ratio $\operatorname{Exp}(B)$ & $95 \%$ confidence interval & $p$ value \\
\hline ADHD & .27 & 8.28 & 1.31 & {$[1.09,1.57]$} & .004 \\
Anxiety Disorders & .30 & 11.26 & 1.35 & {$[1.13,1.61]$} & .001 \\
RAD & .98 & 7.39 & 2.66 & {$[1.31,5.40]$} & .007 \\
Mood Disorders & .58 & 29.02 & 1.79 & {$[1.45,2.21]$} & .000 \\
Substance-Related & .22 & .60 & 1.24 & {$[.72,2.15]$} & .427 \\
Disorders & & & 1.10 & {$[.69,1.74]$} & .694 \\
Sleep Disorders & .09 & .16 & &
\end{tabular}




\section{Discussion}

The purpose of the present study was to examine the link between provisional psychiatric diagnoses and potential experiences of early trauma or adversity in children and youth. Overall, the present study suggests that a number of provisional diagnoses are highly related to potential traumatic experiences for children and youth. Below, we review how the findings of this study add to our understanding of the potentially underappreciated effects of trauma, and discuss implications for current clinicians in terms of diagnosis, as well as implications for the broader mental health community as it attempts to move towards a more comprehensive understanding of developmental trauma.

We begin by briefly reviewing the most common provisional diagnoses in our sample and how these diagnoses may relate to a potential underlying developmental trauma formulation. Our findings indicated that girls were more likely to have a provisional diagnosis of mood, sleep, or anxiety disorder; whereas, boys were more likely to carry a provisional diagnosis of ADHD. This finding is a replication of previous research suggesting that young boys are far more likely to receive diagnoses along the externalizing spectrum; whereas, girls are more likely to receive diagnoses of internalizing disorders (e.g., Achenbach, Howell, Quay, Conners, \& Bates, 1991; Dodge, Pettit, \& Bates, 1994; Keiley et al., 2003). Importantly, there are many shared behaviors between various mental health disorders and potential reactions to traumatic events. For example, in ADHD many authors have argued that the observable behaviors that children present with (e.g., difficulty with sustained attention, fidgeting, impulsivity, etc.) are the same behavioral manifestations that we would expect to see for a child who has been exposed to potentially traumatic events (e.g., Cook et al., 2005; Shonkoff et al., 2012; Swingle \& Swingle, 2016; van der Kolk, 2005). ADHD is but one example of a mental health disorder that may be overrepresented in the population due to the lack of appropriate nosological categories for trauma-related symptomatology (D'Andrea et al., 2012).

Next, we turn to the question of whether provisional diagnoses in our sample of children/youth were related to potential traumatic stress. Here, our findings were clear and just as before needed to be separated by sex. First, for boys, results indicated $\mathrm{ADHD}$, anxiety disorders, RAD, mood disorders, and substance-related disorders increased the odds of triggering the interRAI Traumatic Life Events CAP. Second, for girls, results indicated that ADHD, anxiety disorders, RAD, and mood disorders increased the odds of triggering the interRAI Traumatic Life Events CAP. Taken together, these results support previous findings that children and youth who have been exposed to significant levels of trauma (e.g., maltreatment) or adversity often meet the DSM-5 (American Psychiatric Association, 2013) criteria for a number of diagnoses, including: ADHD, conduct disorder, anxiety disorders, communication disorders, and various disorders of attachment, including RAD (Cook et al., 2005). Importantly, these results also highlight a specific need for clinicians to assess for traumatic expe- 
riences when formulating a client's presentation. If we do not ask the question, "What happened to this child/youth?" and instead focus simply on "What is wrong with this child/youth?" we run the risk of over-diagnosing specific mental health disorders and missing the potential underlying causes for their difficulties. Thankfully, as a field our understanding of the fundamental mechanisms related to trauma-related disorders has improved significantly over the past 15 years, and we now have more substantive knowledge related to the evidenced-based strategies for intervention and prevention when working with traumatized children and youth.

\section{Conclusion}

There are a few limitations that are important to mention. The interRAI Traumatic Life Events CAP is triggered for children and youth with actual or suspected experience of trauma. With that being said, there are likely some participants in this study who have experienced adverse events but do not yet have symptoms of traumatic stress. Moreover, the CAP also takes into account a few adverse experiences that likely are not chronic or interpersonal in nature (e.g., serious accident or physical impairment). However, upon further analysis only $1 \%$ of the children and youth in the sample had a potentially traumatic experience that was likely not chronic.

Our results continue to highlight the need for our field to actively consider implementing the proposed diagnosis of Developmental Trauma Disorder. Although not diagnostic in nature, our results strongly suggest that a significant portion of the children/youth seen in various community mental health agencies have been impacted by traumatic events. Clinicians working with children/youth need a way of communicating across service providers to ensure that children/youth who are exposed to potentially traumatic events are assessed and treated for the underlying causes of their behavioral symptoms. Further, given the significant progress and developments evidenced in the literature relating to treating developmental trauma, it is critical that children/youth be appropriately assessed in order to provide the most effective treatments available (van der Kolk, 2005).

\section{Acknowledgements}

The authors sincerely thank the children, youth, parents and service providers who participated in this research.

\section{References}

Achenbach, T. M., Howell, C. T., Quay, H. C., Conners, C. K., \& Bates, J. E. (1991). National Survey of Problems and Competencies among Four-To Sixteen-Year-Olds: Parents' Reports for Normative and Clinical Samples. Monographs of the Society for Research in Child Development, i-130. https://doi.org/10.2307/1166156

American Psychiatric Association (2013). Diagnostic and Statistical Manual of Mental Disorders $\left(D S M-5^{\circledR}\right)$. American Psychiatric Pub. 
Anda, R. F., Brown, D. W., Dube, S. R., Bremner, J. D., Felitti, V. J., \& Giles, W. H. (2008). Adverse Childhood Experiences and Chronic Obstructive Pulmonary Disease in Adults. American Journal of Preventative Medicine, 34, 396-403.

https://doi.org/10.1016/j.amepre.2008.02.002

Baglivio, M. T., Wolff, K. T., Piquero, A. R., \& Epps, N. (2015). The Relationship between Adverse Childhood Experiences (ACE) and Juvenile Offending Trajectories in a Juvenile Offender Sample. Journal of Criminal Justice, 43, 229-241.

https://doi.org/10.1016/j.jcrimjus.2015.04.012

Blaustein, M., Cook, A., Cloitre, M., DeRosa, R., Ford, J., Henderson, M. et al. (2003). Complex Trauma in Children and Adolescents: White Paper from the National Child Traumatic Stress Network Complex Trauma Task Force. Boston: Child Trauma Center. http://www.nctnet.org/nctsn_assests/pdf/edu_materials/ComplexTrauma_All.pdf

Cannon, W. B. (1929). Bodily Changes in Pain, Hunger, Fear and Rage (2nd ed.). New York: Appleton.

Caspi, A., Houts, R. M., Belsky, D. W., Harrington, H., Hogan, S., Ramrakha, S. et al. (2016). Childhood Forecasting of a Small Segment of the Population with Large Economic Burden. Nature Human Behaviour, 1, 0005.

https://doi.org/10.1038/s41562-016-0005

Cook, A., Spinazzola, J., Ford, J., Lanktree, C., Blaustein, M., Cloitre, M. et al. (2005). Complex Trauma in Children and Adolescents. Psychiatric Annals, 35, 390-398. https://doi.org/10.3928/00485713-20050501-05

D’Andrea, W., Ford, J., Stolbach. B., Spinazzola, J., \& van der Kolk, B. A. (2012). Understanding Interpersonal Trauma in Children: Why We Need a Developmentally Appropriate Trauma Diagnosis. American Journal of Orthopsychiatry, 82, 187-200. https://doi.org/10.1111/j.1939-0025.2012.01154.x

Dodge, K. A., Pettit, G. S., \& Bates, J. E. (1994). Socialization Mediators of the Relation between Socioeconomic Status and Child Conduct Problems. Child Development, 65, 649-665. https://doi.org/10.2307/1131407

Dube, S. R., Felitti, V. J., Dong, M., Giles, W. H., \& Anda, R. F. (2003). The Impact of Adverse Childhood Experiences on Health Problems: Evidence from Four Birth Cohorts Dating Back to 1900. Preventative Medicine, 37, 268-277.

https://doi.org/10.1016/S0091-7435(03)00123-3

Felitti, V. J., Anda, R. F., Nordenberg, D., Willamson, D. F., Spitz, A. M., Edwards, V. et al. (1998). Relationship of Childhood Abuse and Household Dysfunction to Many of the Leading Causes of Death in Adults: The Adverse Childhood Experiences (ACE) Study. American Journal of Preventative Medicine, 14, 245-258.

https://doi.org/10.1016/S0749-3797(98)00017-8

Keiley, M. K., Lofthouse, N., Bates, J. E., Dodge, K. A., \& Pettit, G. S. (2003). Differential Risks of Covarying and Pure Components in Mother and Teacher Reports of Externalizing and Internalizing Behavior across Ages 5 to 14. Journal of Abnormal Child Psychology, 31, 267-283. https://doi.org/10.1023/A:1023277413027

Koren, D., Arnon, I., \& Klein, E. (1999). Acute Stress Response and Posttraumatic Stress Disorder in Traffic Accident Victims: A One-Year Prospective, Follow-Up Study. American Journal of Psychiatry, 156, 367-373.

Perry, B. D. (1994). Neurobiological Sequelae of Childhood Trauma: Post-Traumatic Stress Disorders in Children. In M. Murburg (Ed.), Catecholamine Function in Post Traumatic Stress Disorder: Emerging Concepts (pp. 233-255). Washington DC: American Psychiatric Press.

Pettit, J. W., Morgan, S., \& Paukert, A. L. (2005). The Stability of Axis I Diagnoses in 
Youth across Multiple Psychiatric Hospitalizations. Child Psychiatry \& Human Development, 36, 53-71. https://doi.org/10.1007/s10578-004-3493-6

Shonkoff, J. P., Garner, A. S., Siegel, B. S., Dobbins, M. I., Earls, M. F., McGuinn, L. et al. (2012). The Lifelong Effects of Early Childhood Adversity and Toxic Stress. Pediatrics, 129, e232-e246. https://doi.org/10.1542/peds.2011-2663

Siegfried, C. B., Blackshear, K., National Child Traumatic Stress Network, with Assistance from the National Resource Center on ADHD: A Program of Children and Adults with Attention-Deficit/Hyperactivity Disorder (CHADD) (2016). Is It ADHD or Child Traumatic Stress? A Guide for Clinicians. Los Angeles, CA \& Durham, NC: National Center for Child Traumatic Stress.

Stewart, S. L., Hirdes, J. P., Curtin-Telegdi, N., Perlman, C. M., McKnight, M., MacLeod, K. et al. (2015a). interRAI Child and Youth Mental Health (ChYMH) Assessment Form and User's Manual: For Use with In-Patient and Community-Based Assessments. Version 9.3. Washington DC: interRAI.

Stewart, S. L., Theall, L. A., Morris, J. N., Berg, K., Björkgren, M., Declercq, A. et al. (2015c). interRAI Child and Youth Mental Health Collaborative Action Plans (CAPs): For Use with the Child and Youth Mental Health Assessment Instrument, Version 9.3. Washington DC: interRAI.

Stewart, S. L., Theall, L., Perry, B., MacLeod, K., Smith, C., Mathias, K. et al. (2015b). Traumatic Life Events CAP. In S. L. Stewart, L. A. Theall, J. N. Morris, K. Berg, M. Björkgren, A. Declercq et al. (Eds.), interRAI Child and Youth Mental Health Collaborative Action Plans (CAPs): For Use with the Child and Youth Mental Health Assessment Instrument, Version 9.3 (pp. 53-63). Washington DC: interRAI.

Swingle, M. K., \& Swingle, P. G. (2016). Are You Sure It's AD (H) D? Biofeedback, 44, 35-41. https://doi.org/10.5298/1081-5937-44.1.05

Van der Kolk, B. A. (2005). Developmental Trauma Disorder: Toward a Rational Diagnosis for Children with Complex Trauma Histories. Psychiatric Annals, 35, 401-408. https://doi.org/10.3928/00485713-20050501-06 